$\underline{\text { Preprint typeset in JHEP style. - HYPER VERSION }}$

LPTENS-02-55

hep-th/0207060

\title{
Cosmological evolution with brane-bulk energy exchange
}

\author{
E. Kiritsis ${ }^{1,2,4}$, G. Kofinas ${ }^{1}$, N. Tetradis ${ }^{3}$, T.N. Tomaras ${ }^{1,4}$ and V. Zarikas ${ }^{4}$ \\ (1) Department of Physics and Institute of Plasma Physics, University of Crete, \\ 71003 Heraklion, GREECE \\ (2) Laboratoire de Physique Théorique de l'Ecole Normale Supérieure \\ 24 rue Lhomond, Paris, CEDEX 05, F-75231, FRANCE \\ (3) Department of Physics, University of Athens, 15771 Zographou, GREECE \\ (4) Foundation of Research and Technology, Hellas, 71110 Heraklion, GREECE \\ E-mail: kiritsis@physics.uoc.gr, kofinas@physics.uoc.gr, \\ tetradis@cc.uoa.gr, tomaras@physics.uoc.gr, vzarikas@ics.forth.gr
}

\begin{abstract}
The consequences for the brane cosmological evolution of energy exchange between the brane and the bulk are analyzed in detail, in the context of a non-factorizable background geometry with vanishing effective cosmological constant on the brane. A rich variety of brane cosmologies is obtained, depending on the precise mechanism of energy transfer, the equation of state of brane-matter and the spatial topology. An accelerating era is generically a feature of our solutions. In the case of low-density flat universe more dark matter than in the conventional FRW picture is predicted. Spatially compact solutions are found to delay their re-collapse.
\end{abstract}




\section{Contents}

1. Introduction 1

2. The model 3

3. Special solutions 6

3.1 "Mirage" radiation for energy outflow 7

3.2 Accelerating solutions 8

3.3 The case of radiation for energy influx 10

3.4 Non-flat solutions 11

4. General treatment of the outflow/influx equations 12

4.1 General analysis of the equations 13

4.2 Solutions with $\rho \approx 0$

4.3 Fixed points 17

5. Conclusions 18

\section{Introduction}

The idea that we might be living inside a defect, embedded in a higher dimensional space has already a long history. Concerning the nature of the defect, a solitonic codimension one or higher topological object was proposed [1] in the context of an ordinary higher dimensional gauge field theory, coupled [2] or not to gravity. It was soon realized, however, that, in contrast to scalar and spin-1/2 fields, it would be difficult to confine gauge fields on such an object. Various interesting ideas and scenaria were studied [3], which have not yet given a fully satisfactory picture. In connection with the topology and the size of the bulk space on the other hand, the popular choice was that the extra dimensions are compact, with size of order $\mathcal{O}\left(M_{P l}^{-1}\right)$. It was argued in [4], that in the context of the heterotic string with supersymmetry broken à la Scherk-Schwarz this should not be true anymore. The scale of supersymmetry breaking is tied to the size of internal dimensions, and a desirable supersymmetry breaking scale of a few $\mathrm{TeV}$ implies an extra dimension of about $10^{-16} \mathrm{~cm}$. Despite difficulties to build a realistic model based on these ideas, the scenario was taken seriously and analyzed further for its phenomenological consequences [5]. 
The situation is drastically different in the context of type-I string theory [6]. A few developments led to an exciting possibility and renewed interest in the whole idea. First, with the discovery of the D-branes as an essential part of the "spectrum" in type-I string theory, one could conjecture that we inhabit such a D-brane embedded in a ten-dimensional bulk. The usual solitonic defect of field theory was thus replaced by an appropriate collection of D-branes, which by construction confine the gauge fields [7], together with all the ingredients of the standard model. All known matter and forces lie on our brane world [8, 9], with the exception of gravity, which acts in the bulk space as well. It was, however, pointed out [10], that for Kaluza-Klein extra dimensions the gravitational force on the brane was consistent with all laboratory and astrophysical experimental data, as long as the extra dimensions were smaller than a characteristic scale. This led to the exciting possibility of two extra dimensions in the sub-millimeter range. Furthermore, it was demonstrated in the context of an appropriate effective five-dimensional theory of gravity, that once we take into account the back reaction of the brane energy-momentum onto the geometry of space-time, the graviton is effectively confined on the brane and Newton's law is reproduced to an excellent accuracy at large distances, even with a non-compact extra dimension 11 .

At the same time, the analysis of the cosmological consequences of the above hypotheses attracted considerable interest. The first step was taken in [12, where the evolution of perfect fluid matter on the brane was studied, with no reference to the bulk dynamics and, therefore, no energy transfer between the brane and the bulk. Alternatively, a bulk-based point of view was adopted in [13], where the cosmology induced on a moving 3-brane in a static Schwarzschild-AdS background $_{5}$ was studied, and a general interpretation of cosmology on moving branes, together with the idea of "mirage" cosmology, were presented in [14. The equivalence of the two approaches was demonstrated in [15]. It was also pointed out that branes provide natural mechanisms for a varying speed of light [16].

Energy-exchange between the brane and the bulk should in principle be included in any realistic cosmological scenario, and its effects have been studied in detail in the context of flat compact extra dimensions [17]. The role of energy-exchange on brane cosmology in the case of non-factorizable extra dimensions has not yet been investigated extensively [18, 19], even though the importance of energy outflow from the brane has already been demonstrated in the context of a Randall- Sundrum configuration with additional gravity induced on the brane [19].

The present paper is an attempt towards a more complete analysis of the cosmic evolution of the brane in the presence of energy flow into or from the bulk. Our aim is to generalize the picture in bulk AdS (bulk gravity plus cosmological constant) by considering a general bulk theory (that includes gravity). There may be more bulk fields and more general bulk-brane couplings. Although this can be formulated by the standard action principle and the relevant exact equations derived and studied 
we have opted in this paper for a short-cut. We are analyzing the regime where the bulk energy, at the brane position, can be consistently neglected from the equations. Moreover, we parameterize appropriately the energy exchange as a specific power of the matter density of the brane. Although there are other valid parameterizations, we opted for this one, motivated by our previous work in [19] where we analyzed the out-flow of energy from the brane due to graviton radiation in the presence of induced gravity. Standard cross section calculations give an out-flow that is a function of the temperature and other fundamental constants of the theory. If one re-expresses the temperature in terms of the running density using the cosmological equations we obtain a rate of flow that is a power of the density, with a dimension-full coefficient that depends on fundamental constants and initial energy densities. This is valid typically for a whole era, that is, piecewise in the cosmological evolution.

In a given theory, with a specific bulk content and brane-bulk couplings, the exponent of the density as well as the coefficient are calculable functions of the coupling constants of the Lagrangian as well as initial densities. It is also obvious that if the bulk theory is approximately conformal the most general form of rate of outflow will be polynomial in the density. Our analysis is "phenomenological" and we parameterize different theories with different forms for the energy in/out-flow.

The paper is organized in five sections of which this introduction is the first. In section 2 the framework of our work is described and the approximations on the brane-bulk exchange are presented. The effective equations for the analysis of the brane cosmology are derived. Section 3 contains several interesting characteristic solutions of the brane cosmology, while an exact treatment of the influx/outflow equations, relevant for a wide range of potentially realistic applications, is presented in section 4. Our results are summarized and the prospects for further research along these lines are discussed in the final section.

\section{The model}

We shall be interested in the model described by the action

$$
S=\int d^{5} x \sqrt{-g}\left(M^{3} R-\Lambda+\mathcal{L}_{B}^{\text {mat }}\right)+\int d^{4} x \sqrt{-\hat{g}}\left(-V+\mathcal{L}_{b}^{\text {mat }}\right),
$$

where $R$ is the curvature scalar of the five-dimensional metric $g_{A B}, A, B=0,1,2,3,5$, $\Lambda$ is the bulk cosmological constant, and $\hat{g}_{\alpha \beta}$, with $\alpha, \beta=0,1,2,3$, is the induced metric on the 3 -brane. We identify $(x, z)$ with $(x,-z)$, where $z \equiv x_{5}$. However, following the conventions of $[1]$ we extend the bulk integration over the entire interval $(-\infty, \infty)$. The quantity $V$ includes the brane tension as well as quantum contributions to the four-dimensional cosmological constant.

We consider an ansatz for the metric of the form

$$
d s^{2}=-n^{2}(t, z) d t^{2}+a^{2}(t, z) \gamma_{i j} d x^{i} d x^{j}+b^{2}(t, z) d z^{2}
$$


where $\gamma_{i j}$ is a maximally symmetric 3-dimensional metric. We use $k=-1,0,1$ to parameterize the spatial curvature.

The non-zero components of the five-dimensional Einstein tensor are

$$
\begin{aligned}
G_{00}= & 3\left\{\frac{\dot{a}}{a}\left(\frac{\dot{a}}{a}+\frac{\dot{b}}{b}\right)-\frac{n^{2}}{b^{2}}\left(\frac{a^{\prime \prime}}{a}+\frac{a^{\prime}}{a}\left(\frac{a^{\prime}}{a}-\frac{b^{\prime}}{b}\right)\right)+k \frac{n^{2}}{a^{2}}\right\}, \\
G_{i j}= & \frac{a^{2}}{b^{2}} \gamma_{i j}\left\{\frac{a^{\prime}}{a}\left(\frac{a^{\prime}}{a}+2 \frac{n^{\prime}}{n}\right)-\frac{b^{\prime}}{b}\left(\frac{n^{\prime}}{n}+2 \frac{a^{\prime}}{a}\right)+2 \frac{a^{\prime \prime}}{a}+\frac{n^{\prime \prime}}{n}\right\} \\
& +\frac{a^{2}}{n^{2}} \gamma_{i j}\left\{\frac{\dot{a}}{a}\left(-\frac{\dot{a}}{a}+2 \frac{\dot{n}}{n}\right)-2 \frac{\ddot{a}}{a}+\frac{\dot{b}}{b}\left(-2 \frac{\dot{a}}{a}+\frac{\dot{n}}{n}\right)-\frac{\ddot{b}}{b}\right\}-k \gamma_{i j}, \\
G_{05}= & 3\left(\frac{n^{\prime}}{n} \frac{\dot{a}}{a}+\frac{a^{\prime}}{a} \frac{\dot{b}}{b}-\frac{\dot{a}^{\prime}}{a}\right), \\
G_{55}= & 3\left\{\frac{a^{\prime}}{a}\left(\frac{a^{\prime}}{a}+\frac{n^{\prime}}{n}\right)-\frac{b^{2}}{n^{2}}\left(\frac{\dot{a}}{a}\left(\frac{\dot{a}}{a}-\frac{\dot{n}}{n}\right)+\frac{\ddot{a}}{a}\right)-k \frac{b^{2}}{a^{2}}\right\} .
\end{aligned}
$$

Primes indicate derivatives with respect to $z$, while dots derivatives with respect to $t$.

The five-dimensional Einstein equations take the usual form

$$
G_{A C}=\frac{1}{2 M^{3}} T_{A C},
$$

where $T_{A C}$ denotes the total energy-momentum tensor.

Assuming a perfect fluid on the brane and, possibly an additional energy-momentum $\left.T_{C}^{A}\right|_{m, B}$ in the bulk, we write

$$
\begin{aligned}
T_{C}^{A} & =\left.T_{C}^{A}\right|_{\mathrm{v}, b}+\left.T_{C}^{A}\right|_{m, b}+\left.T_{C}^{A}\right|_{\mathrm{v}, B}+\left.T_{C}^{A}\right|_{m, B} \\
\left.T_{C}^{A}\right|_{\mathrm{v}, b} & =\frac{\delta(z)}{b} \operatorname{diag}(-V,-V,-V,-V, 0) \\
\left.T_{C}^{A}\right|_{\mathrm{v}, B} & =\operatorname{diag}(-\Lambda,-\Lambda,-\Lambda,-\Lambda,-\Lambda) \\
\left.T_{C}^{A}\right|_{\mathrm{m}, b} & =\frac{\delta(z)}{b} \operatorname{diag}(-\rho, p, p, p, 0),
\end{aligned}
$$

where $\rho$ and $p$ are the energy density and pressure on the brane, respectively. The behavior of $\left.T_{C}^{A}\right|_{m, B}$ is in general complicated in the presence of flows, but we do not have to specify it further at this point.

We wish to solve the Einstein equations at the location of the brane. We indicate by the subscript o the value of various quantities on the brane. Integrating equations (2.3), (2.4) with respect to $z$ around $z=0$ gives the known jump conditions

$$
\begin{aligned}
& a_{o^{+}}^{\prime}=-a_{o^{-}}^{\prime}=-\frac{1}{12 M^{3}} b_{o} a_{o}(V+\rho) \\
& n_{o^{+}}^{\prime}=-n_{o^{-}}^{\prime}=\frac{1}{12 M^{3}} b_{o} n_{o}(-V+2 \rho+3 p) .
\end{aligned}
$$


The other two Einstein equations (2.5), (2.6) give

$$
\begin{gathered}
\frac{n_{o}^{\prime}}{n_{o}} \frac{\dot{a}_{o}}{a_{o}}+\frac{a_{o}^{\prime}}{a_{o}} \frac{\dot{b}_{o}}{b_{o}}-\frac{\dot{a}_{o}^{\prime}}{a_{o}}=\frac{1}{6 M^{3}} T_{05} \\
\frac{a_{o}^{\prime}}{a_{o}}\left(\frac{a_{o}^{\prime}}{a_{o}}+\frac{n_{o}^{\prime}}{n_{o}}\right)-\frac{b_{o}^{2}}{n_{o}^{2}}\left(\frac{\dot{a}_{o}}{a_{o}}\left(\frac{\dot{a}_{o}}{a_{o}}-\frac{\dot{n}_{o}}{n_{o}}\right)+\frac{\ddot{a}_{o}}{a_{o}}\right)-k \frac{b_{o}^{2}}{a_{o}^{2}}=-\frac{1}{6 M^{3}} \Lambda b_{o}^{2}+\frac{1}{6 M^{3}} T_{55},
\end{gathered}
$$

where $T_{05}, T_{55}$ are the 05 and 55 components of $\left.T_{A C}\right|_{m, B}$ evaluated on the brane. Substituting (2.12), (2.13) in equations (2.14), (2.15) one obtains

$$
\begin{gathered}
\dot{\rho}+3 \frac{\dot{a}_{o}}{a_{o}}(\rho+p)=-\frac{2 n_{o}^{2}}{b_{o}} T_{5}^{0} \\
\frac{1}{n_{o}^{2}}\left(\frac{\ddot{a}_{o}}{a_{o}}+\left(\frac{\dot{a}_{o}}{a_{o}}\right)^{2}-\frac{\dot{a}_{o}}{a_{o}} \frac{\dot{n}_{o}}{n_{o}}\right)+\frac{k}{a_{o}^{2}}=\frac{1}{6 M^{3}}\left(\Lambda+\frac{1}{12 M^{3}} V^{2}\right) \\
-\frac{1}{144 M^{6}}(V(3 p-\rho)+\rho(3 p+\rho))-\frac{1}{6 M^{3}} T_{5}^{5} .
\end{gathered}
$$

We are interested in a model that reduces to the Randall-Sundrum vacuum [11 in the absence of matter. In this case, the first term on the right hand side of equation (2.17) vanishes. A new scale $k_{R S}$ is defined through the relations $V=-\Lambda / k_{R S}=$ $12 M^{3} k_{R S}$.

In order to derive a solution that is largely independent of the bulk dynamics, the $T_{5}^{5}$ term on the right hand side of the same equation must be negligible relative to the second one. This is possible if we assume that the diagonal elements of the various contributions to the energy-momentum tensor satisfy the schematic inequality ${ }^{1}$

$$
\left|\frac{\left.T\right|_{\mathrm{m}, B} ^{\text {diag }}}{\left.T\right|_{\mathrm{v}, B} ^{\text {diag }}}\right| \ll\left|\frac{\left.T\right|_{\mathrm{m}, b} ^{\text {diag }}}{\left.T\right|_{\mathrm{v}, b} ^{\text {diag }}}\right| .
$$

Our assumption is that the bulk matter relative to the bulk vacuum energy is much less important than the brane matter relative to the brane vacuum energy. In this case the bulk is largely unperturbed by the exchange of energy with the brane. When the off-diagonal term $T_{5}^{0}$ is of the same order of magnitude or smaller than the diagonal ones, the inequality (2.18) implies $T \ll \rho k_{R S}$.

At this point we find it convenient to employ a coordinate frame in which $b_{o}=n_{o}=1$ in the above equations. This can be achieved by using Gauss normal coordinates with $b(t, z)=1$, and by going to the temporal gauge on the brane

\footnotetext{
${ }^{1}$ Strictly speaking, the left hand side of (2.18) concerns only the 55 components of the bulk contributions to the energy-momentum tensor. The other components do not appear in equations (2.16), (2.17) and do not affect the cosmological evolution on the brane.
} 
with $n_{o}=1$. The assumptions for the form of the energy-momentum tensor are then specific to this frame ${ }^{2}$

Using $\beta \equiv M^{-6} / 144$ and $\gamma \equiv V M^{-6} / 144$, and omitting the subscript o for convenience in the following, we rewrite equations $(2.16)$ and $(2.17)$ in the equivalent form

$$
\begin{gathered}
\dot{\rho}+3(1+w) \frac{\dot{a}}{a} \rho=-T \\
\frac{\dot{a}^{2}}{a^{2}}=\beta \rho^{2}+2 \gamma \rho-\frac{k}{a^{2}}+\chi+\lambda \\
\dot{\chi}+4 \frac{\dot{a}}{a} \chi=2 \beta\left(\rho+\frac{\gamma}{\beta}\right) T,
\end{gathered}
$$

where $p=w \rho, T=2 T_{5}^{0}$ is the discontinuity of the zero-five component of the bulk energy-momentum tensor, and $\lambda=\left(\Lambda+V^{2} / 12 M^{3}\right) / 12 M^{3}$ the effective cosmological constant on the brane.

In the equations above, Eq. (2.21) is the definition of the auxiliary density $\chi$. With this definition the other two equations are equivalent to the original system (2.16,2.17). As we will see later on, in the special case of no-exchange $(T=0) \chi$ represents the mirage radiation reflecting the non-zero Weyl tensor of the bulk.

The second order equation (2.17) for the scale factor becomes

$$
\frac{\ddot{a}}{a}=-(2+3 w) \beta \rho^{2}-(1+3 w) \gamma \rho-\chi+\lambda .
$$

As mentioned above, in the Randall-Sundrum model the effective cosmological constant $\lambda$ vanishes, and this is the value we shall assume in the rest of the paper.

In the special case of $w=1 / 3$ one may define a new function $\tilde{\chi} \equiv \chi+2 \gamma \rho$. The functions $\tilde{\chi}, \rho$ and $a$ satisfy equations (2.20) to (2.23) with $\tilde{\chi}$ in place of $\chi$ and $\gamma=0$. This should be expected, since for $w=1 / 3$ there is no $\gamma$ left in equation (2.17).

\section{Special solutions}

Before presenting exact solutions of equations (2.20)-(2.22), it is instructive to consider a few special cases whose physical content is more transparent.

\footnotetext{
${ }^{2}$ If the vacuum energy dominates over the matter content of the bulk, we expect that the form of the metric will be close to the Randall-Sundrum solution with a static bulk. Thus, we expect (even though we cannot demonstrate explicitly without a full solution in the bulk) that in a generic frame, in which

$$
\left|\frac{\left.T\right|_{\mathrm{m}, B} ^{\text {diag }}}{\left.T\right|_{\mathrm{v}, B} ^{\text {diag }}}\right| \ll 1
$$

we shall have $\dot{b} \simeq 0$. Then the transformation that sets $b=1$ is not expected to modify significantly the energy-momentum tensor.
} 
We concentrate on the low-density region, in which $\rho \ll \gamma / \beta$. In this case we may ignore the term $\beta \rho^{2}$ in the above equations compared to $\gamma \rho$. As a result, equations (2.20) $-(2.22)$ can be written as

$$
\begin{gathered}
\dot{\rho}+3(1+w) H \rho=-T \\
H^{2}=\left(\frac{\dot{a}}{a}\right)^{2}=2 \gamma \rho+\chi-\frac{k}{a^{2}} \\
\dot{\chi}+4 H \chi=2 \gamma T .
\end{gathered}
$$

The cosmological evolution is determined by three initial parameters $\left(\rho_{i}, a_{i}, \chi_{i}\right.$, or alternatively $\left.\rho_{i}, a_{i}, \dot{a}_{i}\right)$, instead of the two $\left(\rho_{i}, a_{i}\right)$ in conventional cosmology. The reason is that the generalized Friedmann equation (2.21) (or 3.2) is not a first integral of the Einstein equations because of the possible energy exchange between the brane and the bulk.

\section{1 "Mirage" radiation for energy outflow}

Let us consider first the case $T>0$, for which there is flow of energy out of the brane. If the brane matter is radiation dominated with $p=\rho / 3$, equations (3.1), (3.3) have an exact solution independently of the explicit form of $T$ :

$$
\rho+\frac{\chi}{2 \gamma}=\left(\rho_{i}+\frac{\chi_{i}}{2 \gamma}\right) \frac{a_{i}^{4}}{a^{4}}
$$

and

$$
H^{2}=\left(2 \gamma \rho_{i}+\chi_{i}\right) \frac{a_{i}^{4}}{a^{4}}-\frac{k}{a^{2}} .
$$

Assume that initially $\chi_{i}=0$. It is clear that the effect of the radiation on the expansion does not disappear even if it decays during the cosmological evolution: the Hubble parameter of equation (3.5) is determined by the initial value of the energy density, diluted by the expansion in a radiation dominated universe. The real radiation energy density $\rho$, however, falls with time faster than $a^{-4}$.

As a simple example we consider $T=A \rho$ with $A>0$. Then equation (3.1) can be integrated, with the result

$$
\rho=\rho_{i}\left(\frac{a_{i}}{a}\right)^{3(1+w)} e^{-A t},
$$

where we have considered the general case $p=w \rho^{3}$. From equation (3.4) with $\chi_{i}=0, w=1 / 3$ we obtain

$$
\chi=2 \gamma \rho_{i} \frac{a_{i}^{4}}{a^{4}}\left(1-e^{-A t}\right) .
$$

\footnotetext{
${ }^{3}$ Having neglected the term $\beta \rho^{2}$ from equations (3.2) and (3.3), these solutions are valid only if $\beta \rho_{i}^{2} \ll H_{i}^{2}$. As we shall show in subsection (4.2) this condition is eventually satisfied for all solutions in the case of outflow of the form discussed here and $k=0$.
} 
The Hubble parameter, given by equation (3.5) with $\chi_{i}=0$, corresponds to an initial radiation density $\rho_{i}$, further diluted only by the expansion. At late times the expansion is the consequence of a "mirage" effect, arising from the original radiation through $\chi$.

The presence of a "mirage" term is possible even without energy flow. More specifically, equation (3.3) has a solution $\chi=C / a^{4}$ even for $T=0$, and $\chi$ can act as "mirage" radiation. The novel feature for $T \neq 0$ is that the "mirage" effect appears through the decay of real brane matter, even if it was absent in the beginning.

The effect persists even if the decaying matter is not radiation. Consider a more realistic case, in which the brane matter consists of non-relativistic particles with $p=0$, that can decay spontaneously into bulk matter. In this case our previous assumption $T=A \rho$ becomes realistic. The brane energy density is given by equation (3.6) with $w=0$. For $\chi_{i}=0$ we find

$$
\chi=\left[2 \gamma A \int_{0}^{t} \frac{a}{a_{i}} e^{-A t} d t\right] \rho_{i} \frac{a_{i}^{4}}{a^{4}}
$$

For $t \rightarrow \infty$, if the scale factor grows more slowly than an exponential, the integral converges and $\chi \sim 1 / a^{4}$. Again, there is a "mirage" effect corresponding to an initial energy density proportional to $\rho_{i}$.

It can be checked easily that, if the decaying matter is only one component of the matter on the brane, the "mirage" effect acts as an additional radiation component at late times. Whether it can affect the density perturbations of real matter and act as hot dark matter is an open question.

For the solution (3.4), the quantity $q \equiv \ddot{a} / a$ equals $-\left(2 \gamma \rho_{i}+\chi_{i}\right) a_{i}^{4} / a^{4} \equiv-\sigma / a^{4}$. For $k \geq 0$, equation (3.5) requires $\sigma>0$ and, therefore, $q<0$. For $k=-1, \sigma$ may be negative, leading to a loitering universe. The radiation case is not realistic for the eternal stage of the universe, but we are going to see in the following a situation with $k=-1, w=0$ and eternal acceleration.

\subsection{Accelerating solutions}

An interesting feature of this framework is the possible presence of accelerating cosmological solutions. We can look for exponential expansion with a constant Hubble parameter $H$, even if the brane content is not pure vacuum energy. This implies that equations (3.1)-(3.3) must have a time-independent solution, without necessarily requiring $w=-1$. The possible fixed points (denoted by $*$ ) of these equations for $k=0$ satisfy

$$
\begin{aligned}
3 H(1+w) \rho_{*} & =-T\left(\rho_{*}\right) \\
H_{*}^{2} & =2 \gamma \rho_{*}+\chi_{*} \\
2 H_{*} \chi_{*} & =\gamma T\left(\rho_{*}\right) .
\end{aligned}
$$


It is clear from equation (3.9) that, for positive matter density on the brane $(\rho>0)$, flow of energy into the brane $(T(\rho)<0)$ is necessary.

The accretion of energy from the bulk depends on the dynamical mechanism that localizes particles on the brane. Its details are outside the scope of our discussion. However, it is not difficult to imagine scenaria that would lead to accretion. If the brane initially has very low energy density, energy can by transferred onto it by bulk particles such as gravitons. An equilibrium is expected to set in if the brane energy density reaches a limiting value. As a result, a physically motivated behavior for the function $T(\rho)$ is to be negative for small $\rho$ and cross zero towards positive values for larger densities. In the case of accretion it is also natural to expect that the energy transfer approaches a negative constant value for $\rho \rightarrow 0$.

The solution of equations (3.9)-(3.11) satisfies

$$
\begin{aligned}
T\left(\rho_{*}\right) & =-\frac{3 \sqrt{\gamma}}{\sqrt{2}}(1+w)(1-3 w)^{1 / 2} \rho_{*}^{3 / 2} \\
H_{*}^{2} & =\frac{1-3 w}{2} \gamma \rho_{*} \\
\chi_{*} & =-\frac{3(1+w)}{2} \gamma \rho_{*} .
\end{aligned}
$$

For a general form of $T(\rho)$ equation (3.12) is an algebraic equation with a discrete number of roots. For any value of $w$ in the region $-1<w<1 / 3$ a solution is possible. The corresponding cosmological model has a scale factor that grows exponentially with time. The energy density on the brane remains constant due to the energy flow from the bulk. Our model is very similar to the steady state model of cosmology [20]. The main differences are that the energy density is not spontaneously generated, and the Hubble parameter receives an additional contribution from the "mirage" field $\chi$ (see equation (3.10)).

The stability of the fixed point (3.9)-(3.11) determines whether the exponentially expanding solution is an attractor of neighboring cosmological flows. If we consider a small homogeneous perturbation around the fixed point $\left(\rho=\rho_{*}+\delta \rho, \chi=\chi_{*}+\delta \chi\right)$ we find that $\delta \rho, \delta \chi$ satisfy

$$
\frac{d}{d t}\left(\begin{array}{c}
\delta \rho \\
\delta \chi
\end{array}\right)=\frac{T\left(\rho_{*}\right)}{\rho_{*}} \mathcal{M}\left(\begin{array}{c}
\delta \rho \\
\delta \chi
\end{array}\right),
$$

where

$$
\begin{aligned}
\mathcal{M} & =\left(\begin{array}{cc}
-\tilde{\nu}+3(1-w) /(1-3 w) & 1 / \gamma(1-3 w) \\
2 \gamma(\tilde{\nu}-2 /(1-3 w)) & -2(1+9 w) /[3(1+w)(1-3 w)]
\end{array}\right) \\
\tilde{\nu} & =\frac{d \ln |T|}{d \ln \rho}\left(\rho_{*}\right)
\end{aligned}
$$


and we have employed the relations (3.9)-(3.14). If the energy flow has a simple form $T(\rho) \propto \rho^{\nu}$, we have $\tilde{\nu}=\nu$. The eigenvalues of the matrix $\mathcal{M}$ are

$$
M_{1,2}=\frac{7+3 w-3 \tilde{\nu}(1+w) \pm \sqrt{24(-3+2 \tilde{\nu})(1+w)+[7+3 w-3 \tilde{\nu}(1+w)]^{2}}}{6(1+w)} .
$$

For $-1<w<1 / 3,0 \leq \tilde{\nu}<3 / 2$ they both have a positive real part. As we have assumed $T(\rho)<0$, the fixed point is stable in this case. We have verified this result by integrating equations (3.1)-(3.3) numerically. The approach to the fixed-point values depends on the sign of the quantity under the square root. If this is negative the energy density oscillates with diminishing amplitude around its fixed-point value.

For $w=-1$ we get the standard inflation only for a value $\rho_{*}$ that is a zero of $T(\rho)$. In this case there is no flow along the fifth dimension and also $\chi_{*}=0$.

\subsection{The case of radiation for energy influx}

In the case of radiation the general solution of equations (3.1)-(3.3) was derived in the previous subsection and is given by equations (3.4), (3.5). The expansion is that of a radiation-dominated universe with constant energy $\left(\chi_{i} /(2 \gamma)+\rho_{i}\right) a_{i}^{4}$ per co-moving volume. The "mirage" energy density is diluted $\sim a^{-4}$.

The explicit dependence on time will be discussed next in the case of flat space $(k=0)$, in which the energy density satisfies

$$
\frac{d \rho}{d t}+\frac{2}{t} \rho=-T(\rho)
$$

If $T(\rho)<0$ for all $\rho$, and the "friction" term in the left hand side becomes suppressed for $t \rightarrow \infty$, we expect an unbounded increase of $\rho$ in this limit. For $\rho \gtrsim \gamma / \beta$ the low energy approximation employed in this section breaks down. The full treatment necessary in this case will be given in the next section.

The actual situation is rather complicated and the details depend crucially on the form of $T(\rho)$. Assuming that $T(\rho)=A \rho^{\nu}$ with $A<0$, the exact solution of equation (3.19) for $\nu \neq 1,3 / 2$ is

$$
\left(\rho \tilde{t}^{2}\right)^{1-\nu}=\left(\rho_{i} \tilde{t}_{i}^{2}\right)^{1-\nu}+\frac{1-\nu}{3-2 \nu}\left(\tilde{t}^{3-2 \nu}-\tilde{t}_{i}^{3-2 \nu}\right),
$$

where $\tilde{t}=|A| t$. For $\nu=1$ the solution is

$$
\rho=\rho_{i} \frac{\tilde{t}_{i}^{2}}{\tilde{t}^{2}} e^{\tilde{t}-\tilde{t}_{i}}
$$

and for $\nu=3 / 2$

$$
\left(\rho \tilde{t}^{2}\right)^{-1 / 2}=\left(\rho_{i} \tilde{t}_{i}^{2}\right)^{-1 / 2}-\frac{1}{2} \ln \left(\frac{\tilde{t}}{\tilde{t}_{i}}\right)
$$


For $0 \leq \nu<1$ we have $\rho \sim \tilde{t}^{1 /(1-\nu)}$ for $\tilde{t} \rightarrow \infty$. For $\nu=1$ the increase of the energy density at large $\tilde{t}$ is exponential moderated by a power. For $1<\nu<3 / 2$ the energy density diverges at a finite time

$$
\tilde{t}_{d}^{3-2 \nu}=\tilde{t}_{i}^{3-2 \nu}+\frac{3-2 \nu}{1-\nu}\left(\rho_{i} \tilde{t}_{i}^{2}\right)^{1-\nu} .
$$

A similar divergence appears for $\nu=3 / 2$. For $\nu>3 / 2$ a divergence occurs if the quantity

$$
D=\left(\rho_{i} \widetilde{t}_{i}^{2}\right)^{1-\nu}-\frac{\nu-1}{2 \nu-3} \widetilde{t}_{i}^{2 \nu-3}
$$

is negative. In the opposite case $\rho \tilde{t}^{2} \rightarrow 1 / D$ for $t \rightarrow \infty$, and the energy density diminishes: $\rho \sim t^{-2}$.

As we discussed earlier, it is physically reasonable that the energy influx should stop at a certain value $\rho_{c r}$, and be reversed for larger energy densities. The dynamical mechanism that localizes particles on the brane cannot operate for arbitrarily large energy densities. This modifies the solutions above that predict an unbounded increase of the energy density.

A final observation that will be encountered again in the next section is that, despite of the fact that the energy density in most cases increases for large times, it can decrease at the initial stages. This is obvious from eq. (3.19). If at the time $t_{r}$ that the brane enters a radiation dominated era $|T(\rho)| / \rho<2 / t$, the energy density decreases for a certain time.

\subsection{Non-flat solutions}

In addition to the analytical special solutions discussed above, we would like to present a few suggestive numerical results concerning the $k= \pm 1$ cases. For $\nu=1$, we substitute (3.6), true for any $A$, into the second order equation (2.17), to obtain

$$
\frac{\ddot{a}}{a}+\frac{\dot{a}^{2}}{a^{2}}+\frac{k}{a^{2}}+(1+3 w) \beta \rho_{1}^{2} \frac{e^{-2 A t}}{a^{6(1+w)}}-(1-3 w) \gamma \rho_{1} \frac{e^{-A t}}{a^{3(1+w)}}=0 .
$$

It is obvious from (3.25) that for outflow with $k=1$ and $w \geq-1 / 3$ the Universe will exhibit eternal deceleration. In particular, in the case of dust, Figure 1 depicts the solution for $a(t)$ of (3.25) for some initial conditions for $a, \rho$. Notice that $a(t)$ after a period of decrease, starts increasing again, thus delaying its re-collapse. Of course, with appropriate initial conditions one also obtains solutions with the standard FRW behavior.

For an open $(k=-1)$ Universe with $A>0, w=0, \nu=1$ we have found numerically a solution where $\rho(t)$ monotonically decreases to zero, while $a(t)$ starts with deceleration, but later on accelerates eternally; more specifically, $q(t) \rightarrow 0^{+}$for $t \rightarrow 0$. Another possibility for $k=-1$ and $A<0, w=0, \nu=1$ allows for a Universe starting with acceleration at infinite densities, which later on, turns to deceleration with $\rho$ approaching a constant value. 


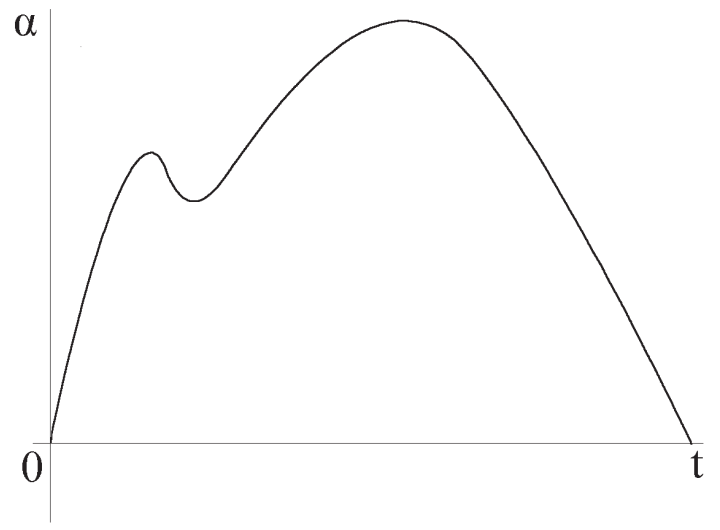

Figure 1: Outflow, $k=+1, w=0, \nu=1$.

\section{General treatment of the out- flow/influx equations}

In this section, we present a few first steps towards an exact analytical treatment of equations (2.20)-(2.22) that govern the cosmological evolution. As we mentioned earlier, we concentrate on the case with zero effective cosmological constant $\lambda$ and investigate the brane cosmological dynamics due to the energy exchange with the bulk.

Some qualitative properties of the exact solutions can be derived from general

arguments:

a) The evolution equations for $T=0$ reduce to those of ref. [12]: (2.20) is the standard energy conservation equation, while (2.22) upon integration leads to the usual mirage radiation term $\chi=C / a^{4}$. If there is no energy exchange between the brane and the bulk $(T=0)$, it can be seen from equation (2.23) that for suitable $C<0$ an acceleration era $(\ddot{a}>0)$ is possible. It is tempting to associate this era with the recently observed cosmic acceleration [21]. It is an open question, however, if the preceding deceleration era (with a dominant contribution $\sim \rho^{2}$ to the Hubble parameter) can accommodate the successes of standard Big-Bang cosmology.

b) For $T \neq 0$, a suitable negative $\chi$ term can, in principle, produce acceleration (i.e. behave like a positive cosmological constant in equation (2.23)) and at the same time play a role similar to that of a negative cosmological constant in the Hubble expansion (2.21). A consequence of this fact is that, if equations $(2.20)-(2.23)$ are to describe the present-day Universe with $\ddot{a} / a>0$, we must require $\chi<0$. In the asymptotic regime $\rho \ll \gamma / \beta$, this means that, for $k=0$, the matter content of the critical Universe must compensate for the negative contribution from $\chi$. This requires $\Omega_{m}>2$ and $\Omega_{\chi}<-1$. As a result, this scenario predicts more dark energy than conventional FRWL models. Notice, however, that for $k=-1$ these restrictions do not apply. For example, we could have $\Omega_{k}=0.96, \Omega_{\chi}=-0.06$ and $\Omega_{m}=0.1$.

c) In the case of outflow $(T>0)$, it is obvious from the conservation equation (2.20) that $\rho(t)$ is monotonically decreasing for all expanding solutions. Furthermore, if the function $T(\rho)$ vanishes only at $\rho=0, \rho(t) \rightarrow 0$ for $t \rightarrow+\infty$.

d) It is straightforward to argue that for $k=0,+1$ and $w>-1 / 3$, when $\rho>$ $(1-3 w) \gamma /((1+3 w) \beta)$ we have necessarily deceleration. For $k=0,+1, w \geq 1 / 3$ we have deceleration at all times. The absolute upper bound for $\ddot{a} / a$ is at all times $(1-3 w)^{2} \gamma^{2} /(4(1+3 w) \beta)$. These conclusions follow from the fact that the left hand side of equation (2.21) must be positive definite. This implies that $\ddot{a} / a$ lies below the parabola $(1-3 w) \gamma \rho-(1+3 w) \beta \rho^{2}$, which for $w \geq 1 / 3$ is negative definite. Finally, 
one may see from equations (2.21), (2.22) that all expanding $k=0$ solutions with $\rho \rightarrow 0$ for $t \rightarrow+\infty$, will have $\chi \rightarrow 0, \ddot{a} / a \rightarrow 0^{-}$and $\dot{a} / a \rightarrow 0^{+}$, for functions $T(\rho)$ with $T(0)=0$, while for $T(0)<0, \rho \rightarrow 0$ is not possible.

\subsection{General analysis of the equations}

Combining equations (2.20) and (2.21) we obtain

$$
a \frac{d \rho}{d a}=-3(1+w) \rho-\epsilon T(\rho)\left(\beta \rho^{2}+2 \gamma \rho-\frac{k}{a^{2}}+\chi\right)^{-1 / 2} .
$$

Similarly, equations (2.22) and (2.21) give

$$
a \frac{d \chi}{d a}=-4 \chi+2 \epsilon \beta\left(\rho+\frac{\gamma}{\beta}\right) T(\rho)\left(\beta \rho^{2}+2 \gamma \rho-\frac{k}{a^{2}}+\chi\right)^{-1 / 2}
$$

where $\epsilon=1$ refers to expansion, while $\epsilon=-1$ to contraction. These two equations form a two-dimensional dynamical system. The function $\chi(\rho)$ is obtained from the equation

$$
\begin{aligned}
& \left(3(1+w) \rho \sqrt{\beta \rho^{2}+2 \gamma \rho-\frac{k}{a^{2}}+\chi}+\epsilon T(\rho)\right) \frac{d \chi}{d \rho} \\
& =4 \chi \sqrt{\beta \rho^{2}+2 \gamma \rho-\frac{k}{a^{2}}+\chi}-2 \epsilon \beta\left(\rho+\frac{\gamma}{\beta}\right) T(\rho) .
\end{aligned}
$$

Note that the equations of contraction are those of expansion with the roles of outflow and influx interchanged. Combining equations (4.3) with (2.23) we derive the following equation for $q(\rho)$ :

$$
\begin{aligned}
& \left.3(1+w) \rho+\epsilon T(\rho) / \sqrt{(1-3 w) \gamma \rho-(1+3 w) \beta \rho^{2}-k a^{-2}-q(\rho)}\right) \frac{d q(\rho)}{d \rho} \\
& +\epsilon(2(1+3 w) \beta \rho-(1-3 w) \gamma) T(\rho) / \sqrt{(1-3 w) \gamma \rho-(1+3 w) \beta \rho^{2}-k a^{-2}-q(\rho)} \\
- & 4 q(\rho)+(1+3 w)(2(2+3 w) \beta \rho-(1-3 w) \gamma) \rho=0 .
\end{aligned}
$$

We shall use this equation below to decide about eras of acceleration and deceleration in the cosmic evolution on the brane. For $|w|<1 / 3, T(\rho)>0$ and $\rho<((1-$ $3 w) \gamma) /(2(2+3 w) \beta)$, it can be seen from the above equation that $q>0$ implies $d q / d \rho>0$. However, in the range $((1-3 w) \gamma) /(2(1+3 w) \beta)<\rho<((1-3 w) \gamma) /((1+$ $3 w) \beta$ ), passage from acceleration to deceleration during expansion is not allowed.

Another form of the system of equations (4.1), (4.2), which will be helpful for the study of fixed points of the system, can be derived by defining $\alpha \equiv \ln a$ and $Q \equiv q+k e^{-2 \alpha}$. Then

$$
\frac{d \rho}{d \alpha}=R(\rho, Q) \quad, \quad \frac{d Q}{d \alpha}=2 k e^{-2 \alpha}+S(\rho, Q),
$$


where

$$
\begin{aligned}
R(\rho, Q)= & -3(1+w) \rho-\epsilon T\left(-(1+3 w) \beta \rho^{2}+(1-3 w) \gamma \rho-Q\right)^{-1 / 2} \\
S(\rho, Q)= & (1+3 w)(2(2+3 w) \beta \rho-(1-3 w) \gamma) \rho-4 Q \\
& +\epsilon T(2(1+3 w) \beta \rho-(1-3 w) \gamma)\left(-(1+3 w) \beta \rho^{2}+(1-3 w) \gamma \rho-Q\right)^{-1 / 2} .
\end{aligned}
$$

When the 3-metric is flat $(k=0)$, equation (4.3) becomes an autonomous equation, which, in principle, may be solved for $\chi(\rho)$. We define

$$
Y(\rho) \equiv \rho^{-\frac{2}{3(1+w)}} \sqrt{\beta \rho^{2}+2 \gamma \rho+\chi}+\frac{\epsilon}{3(1+w)} \rho^{-\frac{5+3 w}{3(1+w)}} T(\rho),
$$

in terms of which equation (4.3) translates to

$$
\begin{aligned}
Y \frac{d Y}{d \rho} & +\frac{\epsilon}{3(1+w)} \rho^{-\frac{8+6 w}{3(1+w)}}\left(\frac{7+3 w}{3(1+w)} T-\rho \frac{d T}{d \rho}\right) Y-\frac{2}{27(1+w)^{3}} \rho^{-\frac{13+9 w}{3(1+w)}} T^{2} \\
& +\left(\frac{1-3 w}{3(1+w)} \gamma-\frac{1+3 w}{3(1+w)} \beta \rho\right) \rho^{-\frac{4}{3(1+w)}}=0
\end{aligned}
$$

We shall simplify our discussion by considering energy transfers of the form

$$
\epsilon T(\rho)=A \rho^{\nu},
$$

with $\nu$ an arbitrary real parameter. The brane-bulk energy exchange in the real world may be much more complicated. For example, it may be a sum of terms of the above form.

With the change of variable

$$
\bar{\rho}=\rho^{\nu-\frac{5+3 w}{3(1+w)}},
$$

the equation for $Y$ becomes

$$
\begin{aligned}
Y \frac{d Y}{d \bar{\rho}} & -B Y \\
& +\frac{1}{5+3 w-3 \nu(1+w)}\left(\frac{2 A^{2}}{9(1+w)^{2}} \bar{\rho}+(1+3 w) \beta \bar{\rho}^{-r}-(1-3 w) \gamma \bar{\rho}^{-s}\right)=0
\end{aligned}
$$

where $B \equiv A(7+3 w-3 \nu(1+w)) /(3(1+w)(5+3 w-3 \nu(1+w))), r \equiv(7+9 w-$ $3 \nu(1+w)) /(5+3 w-3 \nu(1+w))$ and $s \equiv(4+6 w-3 \nu(1+w)) /(5+3 w-3 \nu(1+w))$.

In the cases with $-1<w \leq 1 / 3, \nu \leq(4+6 w) /(3(1+w))$, or $w \geq 1 / 3$, $\nu<(5+3 w) /(3(1+w))$, or $-1<w \leq-1 / 3, \nu>(5+3 w) /(3(1+w))$, or $w \geq-1 / 3$, $\nu \geq(7+9 w) /(3(1+w))$, we have $r, s \geq 0$. In the case $w \geq 1 / 3,(5+3 w) /(3(1+w))<$ $\nu \leq 3 / 2$, we have $r, s \leq-1$. In all these cases, the behavior $\rho \approx 0$, if such a region exists, is given by the following approximation of equation (4.12):

$$
Y \frac{d Y}{d \bar{\rho}}-B Y+\frac{2 A^{2}}{9(1+w)^{2}(5+3 w-3 \nu(1+w))} \bar{\rho}=0 .
$$




\subsection{Solutions with $\rho \approx 0$}

Case 1: For $\nu=1$ (and thus $w>-1 / 3$ ), as in the case of unstable matter on the brane decaying into the bulk, the general solution of (4.13) is 22

$$
\left(\bar{\rho}-\frac{3(1+w)}{A} Y\right) \exp \left(\frac{\bar{\rho}}{\bar{\rho}-\frac{3(1+w)}{A} Y}\right)=-\frac{3(1+w)}{A} \kappa .
$$

Equivalently, in terms of the function $\chi$, the above solution takes the form

$$
\sqrt{\beta \rho^{2}+2 \gamma \rho+\chi} \exp \left(-\frac{A}{3(1+w)} \frac{1}{\sqrt{\beta \rho^{2}+2 \gamma \rho+\chi}}\right)=\kappa \rho^{\frac{2}{3(1+w)}}
$$

where $\kappa$ is a non-negative integration constant. Using (2.21) we may rewrite the above as

$$
|H| e^{-\frac{A}{3(1+w)} \frac{1}{|H|}}=\kappa \rho^{\frac{2}{3(1+w)}} .
$$

Equation (4.16), as is suggested by its independence on $\beta$ and $\gamma$, is valid only as long as the $\beta$ and $\gamma$ terms in (4.12) are negligible compared to the linear term inside the parenthesis. In the case of outflow this is not a constraint, since $\rho$ is monotonically decreasing towards zero. So, at late times all solutions are correctly described by (4.16).

From equation (4.14) we conclude that for $A<0$ (influx), $\nu=1, w>-1 / 3$, any solution $\rho(t)$ with $\kappa>0$ is bounded from below by some positive value. This implies that the point $(\rho=0, q=0)$ cannot be an attractor. Furthermore, using (4.16) and (2.17) one may show that for $w \neq 1 / 3 q \simeq(1-3 w) \gamma \rho$, i.e. all curves near the origin

of the $(q, \rho)$ plane have a fixed slope. This will be confirmed numerically in Figure 3 below. Also, $|H| \ll \rho / M^{3}$.

In the case of outflow, on the other hand, equation (4.16) implies that for $\nu=$ $1, w>-1 / 3$, we have $|H| \rightarrow 0$ for $\rho \rightarrow 0$. Equation (4.16) implies that $\rho \ll|q|$ and in addition that $\rho / q \rightarrow-\infty$ as $\rho \rightarrow 0$, in agreement with the case depicted in Figure 2. Using (2.20)-(2.22) it is straightforward to show that

$$
\frac{d H^{2}}{d \alpha}+4 H^{2}-2(1-3 w) \gamma \rho+2(1+3 w) \beta \rho^{2}=0,
$$

which combined with (4.16) leads to

$$
|H|=\frac{c_{1}}{a^{2}}
$$

or equivalently to

$$
a^{2}(t)=2 c_{1} t+c_{2}
$$

with $c_{1}$ and $c_{2}$ integration constants. Using this expression of $H(t)$ in (4.16), we obtain

$$
\rho(t)=(2 \kappa)^{-3(1+w) / 2} \frac{e^{-A\left(t+\frac{c_{2}}{2 c_{1}}\right)}}{\left(t+\frac{c_{2}}{2 c_{1}}\right)^{3(1+w) / 2}} .
$$


Apart from the obvious agreement of the above with the special solution (3.21) (also valid for $A>0$ ), we should like to stress at this point the remarkable fact that the scale factor $a(t)$ of the Brane Universe, for $\nu=1$ and essentially any $w$, behaves at late times as if radiation dominated.

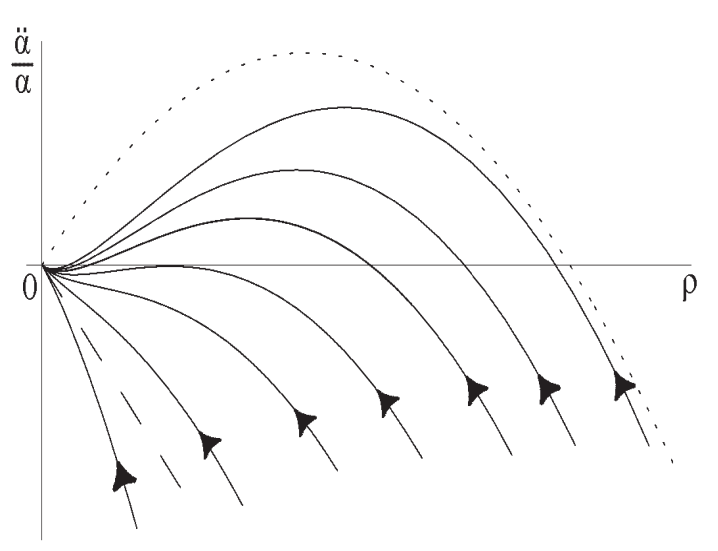

Figure 2: Outflow, $k=0, w=0, \nu=1$. The arrows show the direction of increasing scale factor.
A global phase portrait of $q \equiv \ddot{a} / a$ with respect to $\rho$ during expansion in the outflow case for $k=0, w=0, \nu=1$ is shown in Figure 2. The characteristics of the solutions are in agreement with our conclusions above. One recognizes two families of solutions: The first have $q<0$ for all values of $\rho$, while the second start with a deceleration era for large $\rho$, enter an acceleration era and then return to deceleration for small enough values of $\rho$. This is exactly what should be expected on the basis of the previous analysis. Solutions corresponding to initial conditions with positive $q$ (always under the limiting parabola shown with the dotted line), necessarily had a deceleration era in the past, and are going to end with an eternal deceleration era also. The straight dashed line represents the standard FRW solution.

Case 2: For $\nu \neq 1$, following the same steps as in case 1 , the general solution of (4.13) for $-1<w \leq 1 / 3, \nu \leq(4+6 w) /(3(1+w))$, or $w \geq 1 / 3, \nu<(5+3 w) /(3(1+w))$ is

$$
\kappa_{1}\left|Y-\frac{A}{3(1+w)} \bar{\rho}\right| \frac{5+3 w-3 \nu(1+w)}{2}=\kappa_{2}\left|Y-\frac{2 A}{3(1+w)(5+3 w-3 \nu(1+w))} \bar{\rho}\right|,
$$

where $\left|\kappa_{1}\right|+\left|\kappa_{2}\right|>0$. Using (4.8) and (2.21) this is translated into the implicit solution for $H(\rho)$

$$
\kappa_{1}|H|^{\frac{5+3 w-3 \nu(1+w)}{2}}=\kappa_{2}\left|\rho^{1-\nu}\right| H\left|-\frac{A(\nu-1)}{5+3 w-3 \nu(1+w)}\right| .
$$

A few comments concerning the validity of our approximations are in order at this point. First, we would like to stress that (4.14) and (4.21) were derived and are valid only near "vanishing" $\rho$. Their validity may be questionable in situations with $\rho$ bounded from below. A detailed perturbative analysis of the next order correction to $Y(\rho)$, ensures that the above expressions apply in all cases studied, except for some sets of initial data in the case of energy influx domination $(A<0, \nu<1)$, in which $\rho$ is indeed bounded from below. A similar comment applies to equations (4.16) and (4.22). The translation from $Y$ to $H$ involves equation (4.8), whose possible singular 
nature leads to some further constraints on their validity. Specifically, in the case of $\nu \geq 1$ for both influx and outflow, (4.16) and (4.22) are correct for all solutions with $\rho$ approaching arbitrarily close to zero. For dominant outflow or influx, i.e. $\nu<1$, (4.22) should not be trusted. In these cases, the next order correction to $H$ is of the same order of magnitude as the leading $H$, given in (4.22). Incidentally, the above clarifications remove an apparent contradiction of (4.22) with the general comment $(\mathrm{d})$ in the beginning of this section. Namely, (4.22) implies that for $\nu<1$, $H(\rho)$ tends to a non-vanishing constant as $\rho \rightarrow 0$. This contradicts comment (d) but happens in a region of parameters and initial data for which $(4.22)$ is not reliable.

Finally, for $1<\nu<3 / 2$ it is straightforward to see that $q / \rho \rightarrow-\infty$ and, furthermore, in the case of outflow, the explicit expression for the scale factor is given by (4.19), while the matter density is

$$
\rho(t)=\left(\frac{c_{1}(5+3 w-3 \nu(1+w))}{A(\nu-1)\left(2 c_{1} t+c_{2}\right)}\right)^{1 /(\nu-1)} .
$$

In the case of influx the point $(0,0)$ in the $(\rho-q)$ plane is a repulsor.

\subsection{Fixed points}

Equations (2.16) and (2.17) possess for $k=0$ the obvious fixed point solution $\left(\rho_{*}=\right.$ $\left.0, H_{*}=0\right)$. However, there are more fixed points which may be found by setting $d \rho / d \alpha=d Q / d \alpha=0$ in the system of equations (4.5). This leads to the following relations

$$
\begin{gathered}
(1+3 w) \beta \rho_{*}^{2}-(1-3 w) \gamma \rho_{*}+\frac{2\left|T\left(\rho_{*}\right)\right|^{2}}{9(1+w)^{2} \rho_{*}^{2}}=0, \\
q_{*}=\frac{\left|T\left(\rho_{*}\right)\right|^{2}}{9(1+w)^{2} \rho_{*}^{2}}>0 .
\end{gathered}
$$

It is obvious from the conservation equation (4.1) that during expansion non-trivial fixed points may exist only in the influx case. Their number is determined by the roots of equation (4.24). It can be seen from (4.25) that these solutions are accelerating. From (2.21), (2.22) we find that $H_{*}=\sqrt{q_{*}}$ and $\chi_{*}=-3(1+w)\left(\beta \rho_{*}+\gamma\right) \rho_{*} / 2$.

In order to study the stability of these fixed points we write $\rho(\alpha)=\rho_{*}+\delta \rho(\alpha)$, $q(\alpha)=q_{*}+\delta q(\alpha)$. The resulting linearized equations are

$$
\frac{d}{d \alpha}\left(\begin{array}{l}
\delta \rho(\alpha) \\
\delta q(\alpha)
\end{array}\right)=\frac{9(1+w)^{2} \rho_{*}^{3}}{2 T_{*}^{2}}\left(\begin{array}{ll}
m_{1} m_{2} \\
m_{3} m_{4}
\end{array}\right)\left(\begin{array}{l}
\delta \rho(\alpha) \\
\delta q(\alpha)
\end{array}\right)
$$

where

$$
\begin{aligned}
m_{1}= & 3(1+w)\left((3-\nu)(1+3 w) \beta \rho_{*}+(\nu-2)(1-3 w) \gamma\right) \\
m_{2}= & 3(1+w) \\
m_{3}= & \left((1-3 w) \gamma-2(1+3 w) \beta \rho_{*}\right) \\
& \times\left[(1+3 w)(7+9 w-3 \nu(1+w)) \beta \rho_{*}-(1-3 w)(4+6 w-3 \nu(1+w)) \gamma\right] \\
m_{4}= & -\left(2(1+3 w)^{2} \beta \rho_{*}+(1-3 w)^{2} \gamma\right) .
\end{aligned}
$$


The sign of the real part of the eigenvalues of the matrix appearing above determines the nature of the fixed point. The presence of an imaginary part results in a spiral form for the flows.

As an example, we consider the case with dust $(w=0)$ and $\nu=1$. For $|A| \leq$ $3 \gamma / \sqrt{8 \beta}$, there exist two real positive roots

$$
\rho_{*}^{( \pm)}=\left(1 \pm \sqrt{1-\frac{8 \beta A^{2}}{9 \gamma^{2}}}\right) \frac{\gamma}{2 \beta} .
$$

The root $\rho_{*}^{(+)}$is always a saddle point. Furthermore, for $27 \gamma^{2} /(32 \beta)<A^{2}<$ $9 \gamma^{2} /(8 \beta), \rho_{*}^{(-)}$is a stable node, while for $A^{2}<27 \gamma^{2} /(32 \beta)$, it becomes a counterclockwise stable spiral. For $\beta A^{2} / \gamma^{2} \ll 1$ we have $\rho_{*}^{(-)} \simeq 2 A^{2} /(9 \gamma) \ll \gamma / \beta$ and $\rho_{*}^{(+)} \simeq \gamma / \beta$. In this limit, $\rho_{*}^{(-)}$corresponds to the fixed point (3.12) of the special solutions discussed in section 3 . The eigenvalues of the stability matrix are in agreement with the ones derived there.

The global phase portrait of $q \equiv \ddot{a} / a$ with respect to $\rho$ during expansion for the case $k=0, w=0, \nu=1$ is shown in Figure 3. The presence of the limiting parabola as in the outflow case is apparent. However, new characteristics appear. For example, $\rho_{*}^{(-)}$attracts to eternal acceleration a whole family of solutions which start their evolution at either very low or very high densities. There is another family of solutions which are attracted to acceleration by $\rho_{*}^{(+)}$and which eventually exit to a deceleration era. Finally, there is a family of solutions, near the limiting parabola, which start with acceleration at very low densities, and eventually exit to eternal deceleration, while their density increases monotonically with time because of the influx.

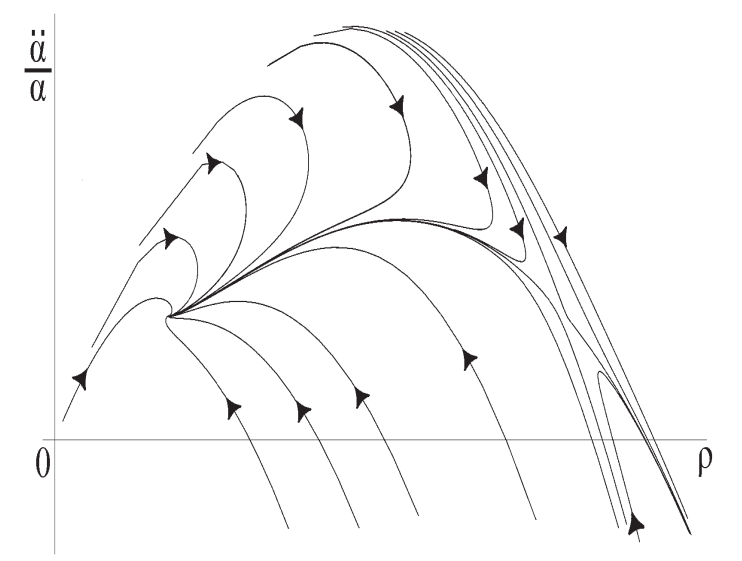

Figure 3: Influx, $k=0, w=0, \nu=1$.
Clearly, for $\nu \neq 1$ one expects a different set of fixed points with varying behaviors around them.

\section{Conclusions}

A rather detailed mathematical analysis of the role of the brane-bulk energy exchange on the evolution of a Brane Universe, adequate for a wide range of potentially realistic implementations, was presented. The effective brane cosmological equations were derived with perfect fluid matter on the brane, constant energymomentum tensor in the bulk and non-vanishing exchange between them. A detailed study of the solutions of these unconventional equations was performed in the case of zero effective cosmological constant on the brane, in order to reduce to the 
Randall-Sundrum vacuum in the absence of matter. The analysis revealed a rather rich variety of possible cosmologies, depending upon the precise form of the exchange term, the topology of 3-space, and the nature of matter on the brane.

A few special but particularly interesting solutions were obtained in the limit of low energy density on the brane. One of these is the exactly solvable case of radiation, where a mirage radiation effect appears through the decay of real brane matter. Another, is a De Sitter fixed point solution, obtained in the case of energy influx, even without pure vacuum energy, which in addition is stable for a wide range of reasonable forms of energy exchange.

An exact treatment of the full set of equations followed. Several generic qualitative bounds were extracted and the asymptotic characteristics of the solutions were studied. In particular, in the low-density regime and for a flat universe, our model predicts additional dark matter compared to the standard cosmological picture. Adopting the physically motivated $T \sim \rho^{\nu}$ power-law form for the energy transfer, we found exact analytical solutions in the low-density regime for almost all values of $\nu$ and of the matter equation of state. Furthermore, the global phase portraits in the density-acceleration plane were obtained numerically, separately for the outflow and the influx cases, for arbitrary values of the density. In the outflow case, one distinguishes two families of solutions: one describing an all-time decelerating universe, and another which describes a Universe with an intermediate accelerating era. The latter starts decelerating, enters an accelerating phase, and finally decelerates again, with the energy density decaying to zero. In the case of influx on the other hand, one generically obtains several fixed point solutions, whose stability analysis was performed. Curiously, all these fixed points correspond to positive acceleration, and their presence implies the existence of solutions with accelerating era. In addition, as one may see in Figure 3, there are solutions that can be purely accelerating, purely decelerating, or with alternating accelerating and decelerating behavior.

A complete description of the evolution of our Brane Universe is still lacking. Several scenaria are possible and their detailed study in the light of observations will determine their viability. For example, a sketchy cosmological evolution could be as follows: The brane is created much hotter than the bulk with a very large energy density somewhere near the limiting parabola. It emits energy to the bulk, and after the initial decelerating period, it acquires an acceleration (presumably, the primordial "inflation"), which for appropriate choice of parameters and initial conditions can be of the order of $\gamma^{2} / \beta$. It quickly cools down and starts decelerating, with energy density much smaller than the one it had initially. Assuming that the energy emission is very fast, it happens with the brane-bulk system out of equilibrium, and one may expect that the brane will supercool and reach a temperature much smaller than the roughly constant temperature of the bulk. As a consequence of the energy influx, the evolution of the brane will be attracted to the stable fixed point, analogous to the $\rho_{*}^{(-)}$discussed in the text. The corresponding $q_{*} \sim \gamma \rho_{*}^{(-)}$will naturally be much 
smaller than its early value, and may fit today's cosmic acceleration 21.

Clearly, the details of scenaria such as the above require further study and many open questions, such as the duration of accelerating periods, the creation of primordial fluctuations, and the compatibility with conventional cosmology at low energy densities, should be addressed. However, we believe that the cosmological evolution in the context we presented here has many novel features, that may provide answers to outstanding questions of modern cosmology.

Some of the cosmological evolutions presented will have a (more general) holographic dual that will be interesting to understand in more detail. This will provide a more controllable picture of the mechanisms advocated in this paper.

\section{Acknowledgments}

This work was partially supported by European Union under the RTN contracts HPRN-CT-2000-00122 and -00131. The work of E. K. was supported by Marie Curie contract MCFI-2001-0214. We would like to thank the referee for helping clarify some points in this paper.

\section{References}

[1] V.A. Rubakov and M.E. Shaposhnikov, Phys. Lett. B125 (1983) 136.

[2] see for instance E. Roessl and M. Shaposhnikov, [arXiv:hep-th/0205320], and references therein

[3] A. Barnaveli and O. Kancheli, Sov. J. Nucl. Phys. 51 (1990) 573; ibid 52 (1990) 576;

G. Dvali and M. Shifman, Phys. Lett. B396 (1997) 64;

N. Tetradis, Phys. Lett. B479 (2000) 265 [arXiv:hep-ph/9908209];

A. Kehagias and K. Tamvakis, Phys. Lett. B504 (2001) 38 [arXiv:hep-th/0010112].

[4] I. Antoniadis, C. Bachas, D. Lewellen and T.N. Tomaras, Phys. Lett. B207 (1988) 441

T. Banks and L.J. Dixon, Nuclear Phys. B307 (1988) 93.

[5] I. Antoniadis, Phys. Lett. B246 (1990) 377;

I. Antoniadis and K. Benakli, Phys. Lett. B 326 (1994) 69 [arXiv:hep-th/9310151];

I. Antoniadis, K. Benakli and M. Quiros, Phys. Lett. B 331 (1994) 313 [arXiv:hepph/9403290]; Phys. Lett. B 460 (1999) 176 [arXiv:hep-ph/9905311].

[6] E. Witten, Nucl. Phys. B471 (1996) 135, [arXiv:hep-th/9602070];

J.D. Lykken, Phys. Rev. D54 (1996) 3693, [arXiv:hep-th/9603133];

C. P. Bachas, In *Goeteborg 1998, Novelties in string theory* 287-293;

C. P. Bachas, JHEP 9811 (1998) 023 [arXiv:hep-ph/9807415].

[7] J. Polchinski, Phys. Rev. Lett. 75 (1995) 4724 [arXiv:hep-th/9510017]. 
[8] see e.g. I. Antoniadis, E. Kiritsis and T.N. Tomaras, Phys. Lett. 487 (2000) 186 [arXiv:hep-ph/0004214]; Fortsch. Phys. 49 (2001) 573 [arXiv:hep-th/0111269].

[9] I. Antoniadis, N. Arkani-Hamed, S. Dimopoulos and G. Dvali, Phys. Lett. B436 (1998) 257 [hep-ph/9804398];

I. Antoniadis and C. Bachas, Phys. Lett. B 450 (1999) 83 [arXiv:hep-th/9812093].

[10] N. Arkani-Hamed, S. Dimopoulos and G. Dvali, Phys. Lett. B429 (1998) 263 [arXiv:hep-ph/9803315].

[11] L. Randall and R. Sundrum, Phys. Rev. Lett. 83 (1999) 3370 [arXiv:hep-th/9905221]; Phys. Rev. Lett. 83 (1999) 4690 [arXiv:hep-th/9906064].

[12] P. Binétruy, C. Deffayet and D. Langlois, Nucl. Phys. B565 (2000) 269 [arXiv:hepth/9905012];

P. Binétruy, C. Deffayet, U. Ellwanger and D. Langlois, Phys. Lett. B477 (2000) 285 [arXiv:hep-th/9910219].

[13] P. Kraus, JHEP 9912 (1999) 011 [arXiv:hep-th:9910149];

D. Ida, JHEP 0009 (2000) 014 [gr-qc/9912002].

[14] A. Kehagias and E. Kiritsis, JHEP 9911 (1999) 022 [arXiv:hep-th/9910174].

[15] S. Mukohyama, T. Shiromizu, K. Maeda, Phys. Rev. D62 (2000) 024028 [hepth/9912287].

[16] E. Kiritsis, JHEP 9910 (1999) 010

[17] L.J. Hall and D.R. Smith, Phys. Rev. D60 (1999) 085008, [arXiv:hep-ph/9904267];

S. Hannestad, Phys. Rev. D64 (2001) 023515, [arXiv:hep-ph/0102290].

[18] C. van de Bruck, M. Dorca, C. J. Martins and M. Parry, Phys. Lett. B 495 (2000) 183 [arXiv:hep-th/0009056];

U. Ellwanger, [arXiv:hep-th/0001126];

A. Hebecker and J. March-Russel, Nucl. Phys. B608 (2001) 375, [arXiv:hep-p h/0103214];

P. Brax, C. van de Bruck and A. C. Davis, JHEP 0110 (2001) 026 [arXiv:hepth/0108215]; D. Langlois, L. Sorbo and M. Rodriguez-Martinez [arXiv:hepth/0206146].

[19] E. Kiritsis, N. Tetradis and T. N. Tomaras, JHEP 0203 (2002) 019 [arXiv:hepth/0202037].

[20] F. Hoyle and J.V. Narlikar, Proc. Royal Soc. A277 (1964) 1; Ann. Phys. 54 (1969) 207.

[21] A.G. Riess et all., Astron. J. 116 (1998) 1009 [arXiv:astro-ph/9805201];

S. Perlmutter et all., Ap. J. 517 (1999) 565 [arXiv:astro-ph/9812133]. 
[22] E. Kamke, Differentialgleichungen Lösungsmethoden und Lösungen, Akademische Verlagsgesellschaft Geest und Portig K.-G., Leipzig 1961. 